\title{
ANALYTIC MODELING OF TRANSIT-TIME DEVICE DRIFT REGIONS WITH FIELD-DEPENDENT TRANSPORT COEFFICIENTS ${ }^{\dagger}$
}

\author{
P. J. MCCLEER, D. E. SNYDER, $\ddagger$ R. O. GRondin and G. I. HADDAD \\ Electron Physics Laboratory. Department of Electrical and Computer Engineering. The University of Michigan, \\ Ann Arbor, MI 48109, U.S.A.
}

(Received 20 December 1979; in revised form 17 March 1980)

\begin{abstract}
An iterative procedure for obtaining two-carrier d.c. solutions in regions of rapidly varying carrier concentration is presented. The procedure uses an analytic solution for the carrier concentrations in a region of linear spatial electric field variation. Field-dependent diffusion and field-dependent velocities are assumed. A single-carrier small-signal model for a drift region with a spatially varying field and field-dependent transport properties is presented. When applied to a BARITT device, results consistent with published experimental data are obtained. The importance of momentum relaxation effects in Si BARITT drift regions is discussed.
\end{abstract}

\section{INTRODUCTION}

Transit-time devices such as IMPATTs, BARITTs and TUNNETTs have been employed as oscillators, amplifiers, frequency multipliers, self-oscillating mixers, optical detectors and video detectors. Although threeterminal devices are replacing them at microwave frequencies, they are likely to be the dominant solid-state device in millimeter-wave applications for many years. Although numerical methods[1-3] are of great utility in modeling these devices they have several important limitations. Obtaining numerical d.c. solutions is difficult in regions of rapidly varying carrier concentrations. Such regions are important in modeling BARITT devices and contact regions. Numerical small-signal solutions typically provide a total device impedance. This is quite satisfactory for oscillator and amplifier applications where the primary concern is with impedance matching to a small-signal negative resistance. However, in mixer and detector applications an equivalent circuit is often more useful. Furthermore, modern automated network analyzers can, by the use of error-correcting procedures [4] and deembedding techniques [5], provide a reasonably good small-signal characterization of these devices at the chip level. If these data are fit to an equivalent circuit whose elements are easily interpreted in terms of device geometry, a powerful experimental characterization of the device is obtained.

In this paper an analytic two-carrier d.c. solution of the standard semiconductor equations under conditions of field-dependent diffusion and velocities is presented. A single-carrier small-signal model including field-depen-

\footnotetext{
†Research sponsored by the Air Force Office of Scientific Research, Air Force Systems Command. USAF, Under Grant No. AFOSR-76-2939C. The United States Government is authorized to reproduce and distribute reprints for Governmental purposes notwithstanding any copyright notation hereon.

$\ddagger$ Present address: Hughes Research Laboratories, Malibu. CA 90265, U.S.A.
}

dent transport coefficients is also presented. This smallsignal model considers only the drift region of the transit-time device and in that sense is an extension of the classic [6-9] transit-time device model of a cathode or injection region connected in series with a drift region. Cathode region parameters differ from device to device and can be obtained from the references. The drift region model developed is expressed as an equivalent circuit whose elements can be easily interpreted in terms of the device geometry. The only other semianalytic smallsignal drift region model[10] which includes both fielddependent diffusion and field-dependent mobilities yields only an overall device impedance. The utility of the model developed here is illustrated by application to a BARITT device.

\section{BASIC SEMICONDUCTOR EQUATIONS}

Transit-time devices typically can be adequately modeled by one-dimensional models. If this dimension is taken to lie along the $x$-axis the basic semiconductor equations are as follows:

Transport equations

$$
\frac{1}{q} J_{n}=-v_{n} n+\frac{\partial}{\partial x}\left(D_{n} n\right)
$$

and

$$
\frac{1}{q} J_{p}=v_{p} p-\frac{\partial}{\partial x}\left(D_{p} p\right) .
$$

Continuity equations

$$
\frac{\partial n}{\partial t}=g_{n}+\frac{1}{q} \frac{\partial}{\partial x}\left(J_{n}\right)
$$

and

$$
\frac{\partial p}{\partial t}=g_{p}-\frac{1}{q} \frac{\partial}{\partial x}\left(J_{p}\right)
$$


Gauss`law

$$
\frac{\partial E}{\partial x}=\frac{q}{\epsilon}\left(N_{D}+p-n\right) .
$$

\section{Current continuity}

$$
\frac{\partial}{\partial x}\left(J_{T}\right)=\frac{\partial}{\partial x}\left(J_{n}+J_{p}+\epsilon \frac{\partial E}{\partial t}\right)=0 .
$$

The variables are defined in Table 1. Since transit-time devices are of great importance in millimeter-wave applications, a cautionary comment should be made at this point. Equations $(3)(6)$ are Maxwell's equations for a one-dimensional system of positive and negative charge carriers. They remain perfectly valid throughout the entire millimeter-wave region provided the constitutive relation $\epsilon E=D_{e}$ between the electric field and electric displacement $D_{e}$ is valid. The transport eqns (1) and (2) however are based on the assumption that the scattering processes occur rapidly with respect to the time variation of the field. The electron distribution function, defined over energy and crystal momentum, will then attain a steady-state value with respect to the instantaneous field. However, the transport equations will become invalid at sufficiently high frequencies[11]. While the exact frequency limitations of (1) and (2) cannot be specified, qualitative results can be obtained for GaAs to the low millimeter-wave region and to somewhat higher frequencies for Si devices. An excellent review of effects not modeled by (1) and (2) is found in [12].

\section{ANALYTIC TWO-CARRIER D.C. SOLUTION OF SPACE-CHARGE} REGIONS

Equations (1)-(6) constitute a coupled set of nonlinear partial differential equations. Their solution can be greatly simplified by assuming that the electric field is a known function of $x$. In many situations where the free carrier densities $n, p$ are much less than the net donor ion density $N_{D}$ the electric field is indeed a known function determined by the integration of Gauss' law with $n=p=0$.

In the more general case, the effects of the mobile space-charge density cannot be ignored. The iterative approach adopted in this paper consists of assuming an electric field profile given by integration of Gauss' law with $n=p=0$. Equations $(1-(4)$ are then solved to determine $p(x)$ and $n(x)$. Gauss' law is used to calculate a new field profile, and the process is repeated until a consistent solution is found. For the drift regions considered in this work. convergence was quite rapid, requiring only 4 to 5 iterations.

The following assumptions are used in the d.c. analysis:

1. The electric field may be approximated as a piecewise linear function.

2. Thermal recombination and generation may be neglected.

3. The Einstein relation

$$
D_{n, p}=\frac{k T}{q} \mu_{n, p}
$$

is assumed to be valid. Here $D_{n, p}$ is the diffusion coefficient for either electrons $\left(D_{n}\right)$ or holes $\left(D_{n}\right), \mu_{n, p}$ is either the electron mobility or the hole mobility, $k$ is Boltzmann's constant, and $T$ is the lattice temperature.

4. In each piecewise linear field region, the following relationship holds:

$$
\left|v_{n, p}\right| \gg\left|\frac{\partial D_{n, p}}{\partial x}\right| .
$$

The transport equations then simplify to

$$
\frac{J_{n}}{q} \simeq-v_{n} n+D_{n} \frac{\partial n}{\partial x}
$$

and

$$
\frac{J_{p}}{q} \simeq v_{p} p-D_{p} \frac{\partial p}{\partial x}
$$

When the Einstein relationship is used, the electron velocity can be written as

$$
v_{n}=-\mu_{n} E=-D_{n} \frac{E}{V_{T}}=-D_{n} \tilde{E}
$$

where $V_{T}$ is the thermal voltage $k T / q$ and $\bar{E}$ is the normalized field $E / V_{T}$. The electron transport equation can then be expressed as

$$
\frac{\mathrm{d} N}{\mathrm{~d} x}+\bar{E} N=\frac{J_{n}}{D_{n}}
$$

Table 1. Variables used in basic semiconductor eqns (1)-16)

\begin{tabular}{ll}
\hline Variable & \multicolumn{1}{c}{ Physical quantity } \\
\hline$D_{n, p}$ & Electron $\left(D_{n}\right)$ and hole $\left(D_{p}\right)$ diffusion coefficients \\
$E$ & Electric field \\
$g_{n, p}$ & Net electron $\left(g_{n}\right)$ and hole $\left(g_{p}\right)$ generation rate \\
$J_{n, p}$ & Electron $\left(J_{n}\right)$ and hole $\left(J_{p}\right)$ electric current densities \\
$J_{T}$ & Total terminal current density \\
$n$ & Electron density \\
$N_{D}$ & Net donor ion density \\
$p$ & Hole density \\
$q$ & Magnitude of the electronic charge \\
$l_{n, p}$ & Electron $\left(v_{n}\right)$ and hole $\left(v_{p}\right)$ drift velocities \\
$\epsilon$ & Electrical permittivity \\
\hline
\end{tabular}


where $N$ is the free electron charge density $n q$. The corresponding hole iransport equation is

$$
\frac{\mathrm{d} P}{\mathrm{~d} x}-\bar{E} P=-\frac{J_{p}}{D_{p}}
$$

where $P$ is the hole charge density $q p$.

5. The field dependence of the diffusion coefficients (and consequently the mobilities) can be represented as the inverse of a polynomial function of $\bar{E}$;

$$
D_{n}=\left(\sum_{k=0}^{K} a_{k} \mid \bar{E}_{\mid}^{k}\right)^{-1}
$$

and

$$
D_{p}=\left(\sum_{m=0}^{M} b_{m}|\bar{E}|^{m}\right)^{-1} .
$$

6. $g_{n}$ and $g_{n}$ are zero. In special cases it is possible to solve the d.c. equations explicitly with nonzero $g_{n}$ and $g_{r}$. In particular, an exponentially varying (optical) generation may be included in the analysis of Si devices where the Trofimenkoff [13] approximation can be used to model the mobilities. Such solutions are necessary in the study of optical detection [14] and optical modulation of microwave diodes $[15,16]$. This optical solution procedure is outlined in Appendix A. In the absence of generation the d.c. continuity equations become

$$
\frac{\mathrm{d} J_{n}}{\mathrm{~d} x}=\frac{\mathrm{d} J_{r}}{\mathrm{~d} x}=0
$$

In a region of linear field variation, $\bar{E}$ can be expressed as

$$
\bar{E}(x)=\bar{E}_{0}+\bar{E}_{1} x
$$

where $\vec{E}_{1}=\mathrm{d} \bar{E} / \mathrm{d} x$. Equations (1I), (13) and (16) yield the relation

$$
\frac{\mathrm{d} N}{\mathrm{~d} x}+\left(\bar{E}_{0}+\bar{E}_{1} x\right) N=J_{n} \sum_{k=0}^{K} a_{k}\left|\bar{E}_{0}+\bar{E}_{1} x\right|^{k}
$$

Since (15) requires that $J_{n}$ be constant, (17) is solved by use of the integrating factor

$$
\exp \left(\int\left(\bar{E}_{0}+\bar{E}_{1} x\right) \mathrm{d} x\right)=\exp \left(\bar{E}_{0} x+\bar{E}_{1} x^{2} / 2+C_{1}\right)
$$

By setting $C_{1}=\bar{E}_{0}{ }^{2} / 2 \bar{E}_{1}$, this factor becomes exp $\left[\xi^{2}\right]$ where

$$
\xi=\frac{\bar{E}_{0}+\bar{E}_{1} x}{\sqrt{ }\left(2 \bar{E}_{1}\right)}
$$

The solution to (17) is then

$$
N(x)=e^{-\xi^{2}}\left(J_{n} \sum_{k=0}^{K} a_{k}\left(2 \bar{E}_{1}\right)^{k / 2} \int|\xi|^{k} e^{\xi^{2}} \mathrm{~d} x+C_{2}\right) .
$$

Although $\xi$ is imaginary for $\bar{E}_{1}<0, N(x)$ remains real. The integral in (20) can be rewritten as

$$
\begin{aligned}
& \int|\xi|^{k} e^{\xi^{2}} \mathrm{~d} x= \pm \sqrt{\left(\frac{2}{\bar{E}_{1}}\right) \int|\xi|^{k} e^{|\xi|^{2}} \mathrm{~d}|\xi|,} \\
& = \pm \vee\left(\frac{2}{\bar{E}_{1}}\right) I_{1}(|\xi|, k), \bar{E}(x) \gtrless 0 .
\end{aligned}
$$

where the plus sign is associated with $\bar{E}(x)>0$. Solutions of $I_{1}(\eta, n)$ for $n=0,1$ along with recursion relations for higher orders of $n$ are given in Appendix B. To simplify evaluation of $C_{2}$ in (20) it is assumed that $\vec{E}$ and therefore $\xi$ does not change sign within the region. This assumption adds no extra complication to the piecewise linear field approximation. When the boundary conditions $N(x=0)=N(0)$ are used and $\xi(x=0)=\xi_{0}$ is denoted, $C_{2}$ can be evaluated and (20) becomes

$$
N(x)=f_{1}\left(\xi_{0}, \xi\right) N(0)+f_{2}\left(\xi_{0}, \xi\right) J_{n},
$$

where

$$
\begin{gathered}
f_{1}\left(\xi_{0}, \xi\right)=\mathrm{e}^{\left.\xi_{0}\right)^{2}-\xi^{2}}, \\
f_{2}\left(\xi_{0}, \xi\right)= \pm 2 \sum_{k=0}^{K} a_{k}\left(2 \bar{E}_{1}\right)^{(k-1) / 2}\left[\mathrm{e}^{-\xi^{2}} I_{1}(|\xi|, k)\right. \\
\left.-\mathrm{e}^{-\xi_{0}{ }^{2}} I_{1}\left(\left|\xi_{0}\right|, k\right) \mathrm{e}^{\xi_{0} T^{2}-\xi^{2}}\right], \bar{E}(x) \gtrless 0 .
\end{gathered}
$$

A similar development for holes yields

$$
P(x)=g_{1}\left(\xi_{0}, \xi\right) P(0)+g_{2}\left(\xi_{0}, \xi\right) J_{p}
$$

where

$$
\begin{gathered}
g_{1}\left(\xi_{0}, \xi\right)=\mathrm{e}^{\xi^{2}-\xi_{0}{ }^{2}} . \\
g_{2}\left(\xi_{0}, \xi\right)= \pm 2 \sum_{m=0}^{M} b_{m}\left(2 \bar{E}_{1}\right)^{(m-1) / 2}\left[\mathrm{e}^{\xi^{2}} I_{2}(|\xi|, m)\right. \\
\left.-\mathrm{e}^{\xi_{0}^{2}} I_{2}\left(\left|\xi_{0}\right|, m\right) \mathrm{e}^{\xi^{2}-\xi_{0}^{2}}\right], \bar{E}(x) \geqslant 0
\end{gathered}
$$

and

$$
I_{2}(\eta, n)=\int^{n} u^{n} \mathrm{e}^{-u^{2}} \mathrm{~d} u
$$

Solutions for $I_{2}(\eta, n)$ are given in Appendix B. The integral functions $I_{1}$ and $I_{2}$ are plotted in Fig. 1 for real values of $\eta$ and various values of $n$.

For a given electric field function, (22) and (23) provide solutions for the mobile space-charge density as linear algebraic functions of $N(0), J_{n}, P(0)$ and $J_{p}$. A collection of $M$ adjacent linear field regions generates a system of $M$ linear equations for $N(x)$ and $M$ linear equations for $P(x)$. This system can be solved iteratively in conjunction with Gauss' law to obtain a self-consistent solution to the d.c. problem.

The diffusion polynomial coefficients are easily determined by a least squares fit to provided data where $a_{0}$ and $b_{0}$ are the inverse of the low-field diffusion coefficients. For Si. good results can be obtained using 


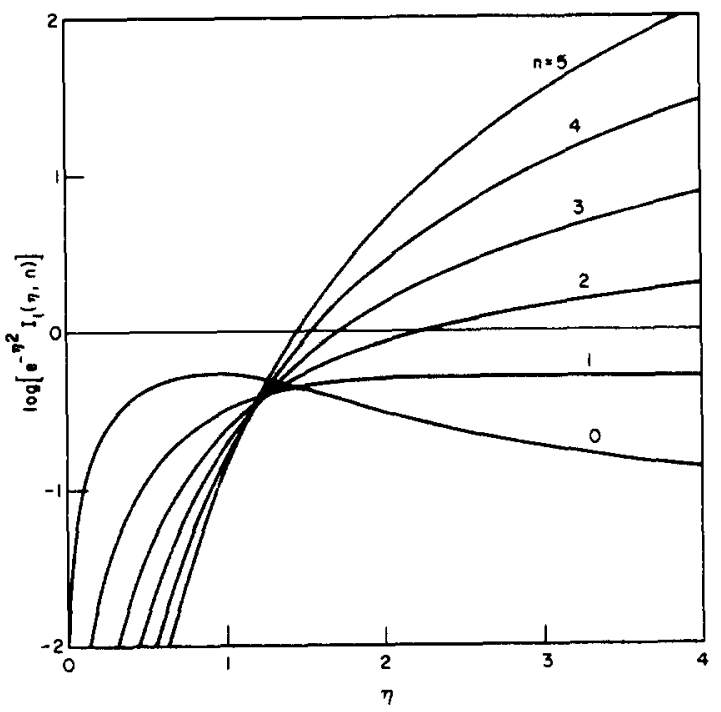

(a)

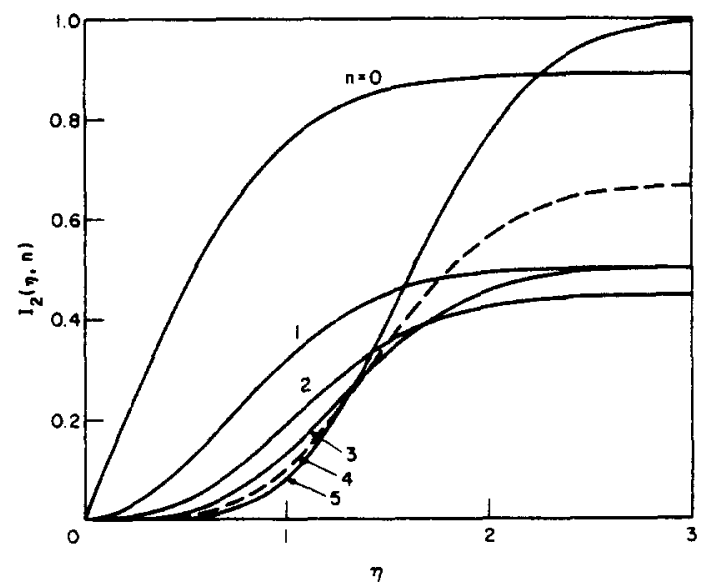

(b)

Fig. 1. (a) The integral function $I_{1}(\eta, n)$. (b) The integral function $I_{2}(\eta, n)$. the Trofimenkoff [13] approximation

$$
\mu=\frac{v_{\mathrm{sat}}}{E_{\mathrm{sat}}+|E|}
$$

or

$$
D=\frac{v_{\text {sat }}}{\bar{E}_{\text {sat }}+|\bar{E}|}
$$

where $v_{\text {sat }}$ is the saturated velocity and $E_{\text {siat }}$ is the electric field at which $v=v_{\text {sat }} / 2$. The polynomial coefficients are then $a_{0}=\bar{E}_{\text {sat }} / v_{\text {sat }}$ and $a_{1}=1 / v_{\text {sat }}$.

For electrons in $\mathrm{GaAs}$ an adequate fit to the mobility relation of Kramer and Mircea[17] can be obtained in the range $0 \leqq E \leqq 20 \mathrm{kV} \mathrm{cm}$ using a fifth-degree polynomial. The polynomial coefficients are given in Table 2 and the resulting velocity-electric field characteristics are compared to those of Kramer and Mircea in Fig. 2.

In performing calculations it is important to note that $\xi^{2}-\xi_{0}^{2}=-\Delta \psi / V_{T}$ where $\Delta \psi$ is the voltage drop across the region considered. As a result, in reverse-biased regions exp $\left[\xi^{2}-\xi_{0}^{2}\right]$ can become extremely large and calculations of $f_{2}\left(\xi_{0}, \xi\right)$ and $g_{2}\left(\xi_{0}, \xi\right)$ can become numerically difficult. For this reason it is convenient to reverse the $x$-axis (and consequently the sign of $\xi$ and $J$ ) when switching from the calculation of $N(x)$ to that of

Table 2. Polynomial coefficients for electron mobility in GaAs

\begin{tabular}{cc}
\hline Coefficient & Value \\
\hline$a_{0}$ & $5.148 \times 10^{-3}$ \\
$a_{1}$ & $-3.835 \times 10^{-8}$ \\
$a_{2}$ & $3.938 \times 10^{-13}$ \\
$a_{3}$ & $2.646 \times 10^{-19}$ \\
$a_{4}$ & $-1.390 \times 10^{-24}$ \\
$a_{5}$ & $9.577 \times 10^{-31}$ \\
\hline
\end{tabular}

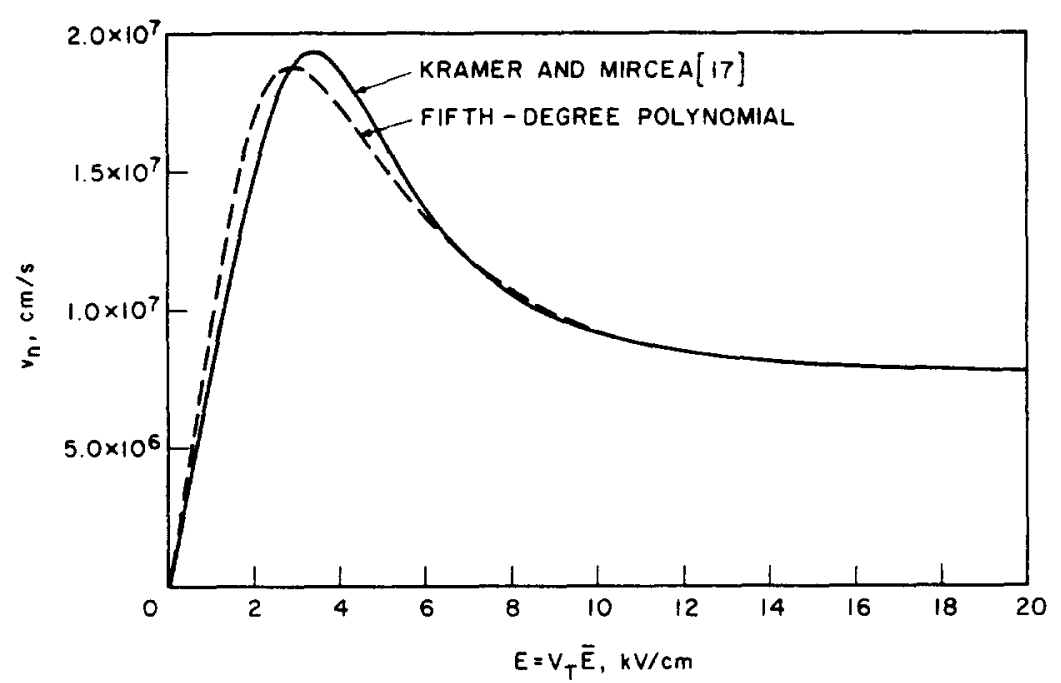

Fig. 2. Comparison of polynomial fit (a) of velocity-field characteristics in GaAs with Kramer and Mircea [17] and (b) $\mu_{0}=7500 \mathrm{~cm}^{2} / \mathrm{V}-\mathrm{s}^{-1}, v_{s}=7.5 \times 10^{6} \mathrm{~cm} / \mathrm{s}^{-1}$, and $E_{v}=4 \times 10^{3} \mathrm{~V} / \mathrm{cm}^{-1}, v_{n}=E\left[\mu_{0}+v_{s}\left(E^{3} / E_{v}^{4}\right)\right] /\left[1+\left(E^{4} / E_{v}^{4}\right)\right]$. Polynomial fit is $v_{n}=\bar{E} / \sum_{n \rightarrow 0}^{3} a_{n} \bar{E}^{n}$ with $a_{n}$ given in Table 2 . 
$P(x) . N(x)$ should always be calculated in the direction of decreasing potential and $P(x)$ in the direction of increasing potential.

\section{SMALL-SIGNAL ANALYSIS OF DRIFT REGIONS}

In this section a single-carrier small-signal model of a drift region containing field-dependent diffusion and field-dependent velocities is presented. Earlier analytic models either assumed saturated velocities with no diffusion [7-9], special cases of field-dependent diffusion or velocities[18-21], or both [10] with no equivalent circuit easily amenable to physical interpretation provided. Here a circuit model is developed which is easily interpreted in terms of the device geometry.

The essential assumption underlying the small-signal analysis is that, for sufficiently small perturbations, linear response theory can be used. Time-varying physical quantities such as voltage and current are expressed as the real part of a complex valued function, e.g.

$$
f(t)=f+\tilde{f} \mathrm{e}^{i \omega t},
$$

where the circumflex denotes the complex phasor coefficient and $f$ is the d.c. component. Higher harmonics are neglected.

By expanding the time-varying quantities in (1)-(6) in the fashion of (27), the basic small-signal equations are obtained. They are as follows:

\section{Transpon equations}

$$
\frac{1}{q} \tilde{J}_{n}=-\tilde{v}_{n} n-v_{n} \tilde{n}+\tilde{D}_{n} \frac{\mathrm{d} n}{\mathrm{~d} x}+D_{n} \frac{\mathrm{d} \tilde{n}}{\mathrm{~d} x}
$$

and

$$
\frac{1}{q} \tilde{J}_{p}=\tilde{v}_{p} p+v_{p} \tilde{p}-\tilde{D}_{p} \frac{\mathrm{d} p}{\mathrm{~d} x}-D_{p} \frac{\mathrm{d} \tilde{p}}{\mathrm{~d} x}
$$

\section{Continuity equations}

$$
i \omega \bar{n}=\frac{1}{q} \frac{\mathrm{d} \bar{J}_{n}}{\mathrm{~d} x}
$$

and

$$
i \omega \bar{p}=-\frac{1}{q} \frac{\mathrm{d} \tilde{J}_{p}}{\mathrm{~d} x}
$$

Gauss' law

$$
\frac{\mathrm{d} \tilde{E}}{\mathrm{~d} x}=\frac{q}{\epsilon}(\tilde{p}-\tilde{n}) .
$$

\section{Current continuity}

$$
\frac{\mathrm{d} \tilde{J}_{T}}{\mathrm{~d} x}=\frac{\mathrm{d}}{\mathrm{d} x}\left[\tilde{J}_{n}+\tilde{J}_{p}+i \omega \epsilon \tilde{E}\right]=0 .
$$

At low frequencies the phasor transport coefficients are the linear term of a Taylor series expansion of the d.c. coefficients. This expansion is sufficiently accurate[22] for use as a high-frequency diffusion coefficient model. For a d.c. field $E_{0}$ and d.c. diffusion coefficient $D_{n, p}$, the phasor coefficient is

$$
\bar{D}_{n, p}=\left.\tilde{E} \frac{\partial D_{n, p}}{\partial E}\right|_{E_{0}} .
$$

It is well known[23] that the phasor mobility varies with frequency as $1 /(1+i \omega \tau)$ for a sinusoidal field with frequency $\omega$ where $\tau$ is the collision process time constant (i.e. the relaxation time). The velocity phasors are then

$$
\tilde{v}_{n}=\left.\frac{\bar{E}}{1+i \omega \tau_{n}} \frac{\partial v_{n}}{\partial E}\right|_{E_{0}}
$$

and

$$
\bar{v}_{p}=\frac{\tilde{E}}{1+i \omega \tau_{p}}-\left.\frac{\partial v_{p}}{\partial E}\right|_{E_{0}} .
$$

The derivatives in (34)-(36) are easily evaluated from the polynomial approximations (13) and (14).

The small-signal solution will use a subdivision of the drift region as illustrated in Fig. 3. The $\Delta x$ must be chosen sufficiently small that the d.c. transport coefficients $D_{n, p}$ and $\mu_{n, p}$ do not vary significantly within the subregion. An approximate solution can now be obtained by replacing all d.c. quantities in the smallsignal eqns (28)-(33) by their spatial average within the subregion of interest. The small-signal equations can then be combined to yield two coupled second-order equations with constant coefficients:

$$
D_{n} \frac{\mathrm{d}^{2} \tilde{n}}{\mathrm{~d} x^{2}}-v_{n} \frac{\mathrm{d} \tilde{n}}{\mathrm{~d} x}-\frac{1}{\epsilon}\left(\tilde{\sigma}_{n}+i \omega \epsilon\right) \tilde{n}+\frac{\tilde{\sigma} n}{\epsilon} \tilde{p}=0
$$

and

$$
D_{p} \frac{\mathrm{d}^{2} \tilde{p}}{\mathrm{~d} x^{2}}-v_{p} \frac{\mathrm{d} \tilde{p}}{\mathrm{~d} x}-\frac{1}{\epsilon}\left(\tilde{\sigma}_{p}+i \omega \epsilon\right) \tilde{p}+\frac{\tilde{\sigma}_{p} \tilde{n}}{\epsilon}=0,
$$

where the small-signal conductivities are

$$
\tilde{\sigma}_{n}=q \tilde{\mu}_{n} n
$$

and

$$
\bar{\sigma}_{p}=q \tilde{\mu}_{p} p .
$$

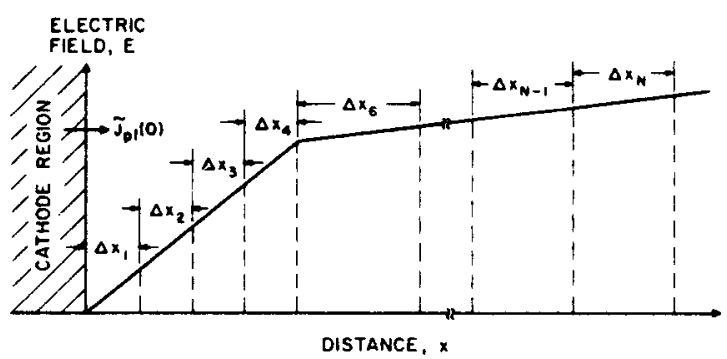

Fig. 3. Division of a typical drift region into $N$ subregions. 
Equations (37) and (38) are the fundamental equations of a space-charge wave solution to the basic small-signal problem (eqns (28)-(33)). They describe the behavior of four coupled traveling space-charge waves, namely, forward and reverse electron and hole waves. By using various approximations a hierarchy of small-signal space-charge wave models can be developed from (37) and (38). The simplest such model, a single wave, is an adequate model for a reverse-biased drift region in most situations. Since the carrier species injected by the cathode is dominant in the drift region, only one of (37) and (38) [(37) for electron injection and (38) for holes] is needed. The coupling term in this equation is neglected. In the absence of coupling the solutions of (37) and (38) are phasor particle densities of the form exp $[\gamma x]$ which describes a traveling space-charge wave. The complex propagation coefficients are:

$$
\gamma_{n}=\frac{1}{2 D_{n}}\left\{v_{n} \pm V\left[v_{n}^{2}+4 D_{n}\left(\tilde{\sigma}_{n}+i \omega \epsilon\right) / \epsilon\right]\right\}
$$

and

$$
\gamma_{p}=\frac{1}{2 D_{p}}\left\{v_{p} \pm V\left[v_{p}^{2}+4 D_{p}\left(\tilde{\sigma}_{p}+i \omega \epsilon\right]\right\}\right.
$$

In (41) and (42) the plus sign before the radical corresponds to space-charge waves traveling against the drift flow. These backward waves are heavily attenuated and may be neglected. The electron and hole density phasors then become

$$
\bar{n}(x)=C_{1} \mathrm{e}^{\gamma_{n}(x)}
$$

and

$$
\tilde{p}(x)=C_{2} \mathrm{e}^{\gamma_{p}(x)},
$$

where $\gamma_{n}$ and $\gamma_{p}$ are obtained from (41)-(42) with the minus sign before the radical.

The single-carrier small-signal model for a hole drift region is now developed. The treatment of an electron drift region is analogous. An equivaient circuit for the subregion is found by substituting (44) into (29). $\tilde{E}$ is replaced in the result by use of (33). Using the boundary condition $\bar{J}_{p}(x=0)=\bar{J}_{p}(0)$ then yields

$$
j_{p}(x)=\tilde{J}_{p}(0) \mathrm{e}^{\gamma_{p} x}+\frac{\tilde{J}_{T} \tilde{\sigma}_{p}}{\bar{\sigma}_{p}+i \omega \epsilon}\left(1-\mathrm{e}^{\gamma_{p} x}\right) .
$$

Since $\tilde{J}_{T}$ is constant it can be spatially averaged over the subregion to yield

$$
\begin{aligned}
\frac{1}{\Delta x} \int_{0}^{\Delta x} \tilde{J}_{T} \mathrm{~d} x & =\tilde{J}_{T} \\
& =\frac{1}{\Delta x} \int_{0}^{\Delta x} \tilde{j}_{p}(x) \mathrm{d} x+\frac{i \omega \epsilon}{\Delta x} \int_{0}^{\Delta x} \tilde{E}(x) \mathrm{d} x .
\end{aligned}
$$

The first integral on the r.h.s. of (46) is a small-signal induced current while the second is a small-signal dis- placement current. Space-charge interaction between the conduction and displacement currents is included in (45). Substituting (45) into (46) yields

$$
\tilde{J}_{T}=\tilde{J}_{p}(0) \Gamma_{m}+\frac{Y}{A} \tilde{V}
$$

where

$$
\begin{gathered}
Y=\frac{\omega C(G+i \omega C)}{\Gamma G+i \omega C}, \\
\Gamma_{m}=\Gamma \frac{Y}{i \omega C^{\prime}}, \\
C=\frac{\epsilon A}{\Delta x}, \\
G=\frac{\tilde{\sigma}_{p} A}{\Delta x}, \\
\Gamma=\frac{1}{\Delta x} \int_{0}^{\Delta x} \mathrm{e}^{\gamma_{p} x} \mathrm{~d} x=\frac{\mathrm{e}^{\gamma_{p} \Delta x}-1}{\gamma_{p} \Delta x}, \\
\tilde{V}=\int_{0}^{\Delta x} \tilde{E}(x) \mathrm{d} x
\end{gathered}
$$

and $A$ is the cross-sectional area of the device.

Equation (47) is represented by the equivalent circuit shown in Fig. 4(a). The impedance of this circuit is

$$
Z=\frac{1}{Y}\left(1-M_{\mathrm{GH}} \Gamma_{m}\right)
$$

In (54) $M_{G H}$ is the Gilden-Hines current fraction[7] defined by

$$
M_{\mathrm{GH}}=\frac{\tilde{j}_{p}(0)}{\tilde{J}_{T}} .
$$

The complex admittance $Y$ can be represented by the equivalent circuit of Fig. $4(\mathrm{~b})$ where $R=1 / G$ is the

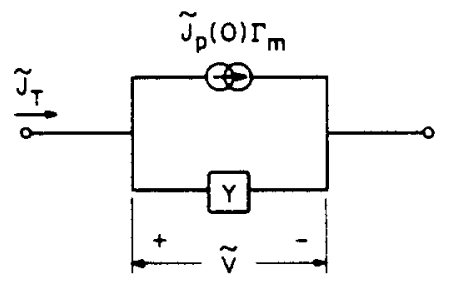

(a)

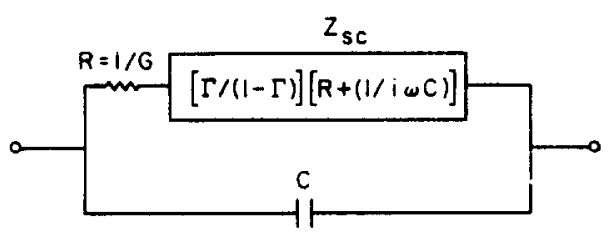

(b)

Fig. 4. (a) Equivalent circuit for a subregion of the drift region and (b) equivalent circuit for admittance $Y$ in Fig. 4(a). 
small-signal bulk resistance of the subregion. The quantity

$$
Z_{s c}=\frac{\Gamma}{1-\Gamma}\left(R+\frac{1}{i \omega C}\right)
$$

will be referred to as the space-charge impedance.

The equivalent circuit for the entire drift region is the series connection of $N$ subregion equivalent circuits (Fig. 5). In the subregion adjacent to the cathode, $M_{\mathrm{GH}}$ is determined solely by the cathode. For the $j$ th subregion $M_{\mathrm{GH}, j}$ is

$$
M_{\mathrm{GH}, j}=M_{\mathrm{GH}, 1} P_{j}+N_{j}
$$

where $M_{\mathrm{GH} .1}$ is the cathode-determined $M_{\mathrm{GH}}$,

$$
P_{j}=\prod_{k=1}^{i-1} \exp \left[\gamma_{k} \Delta x_{k}\right]
$$

and

$$
\begin{aligned}
& N_{j}=\exp \left[-\gamma_{p j} \Delta x_{j}\right] \sum_{k=1}^{-1}\left[\frac{\tilde{\sigma}_{p k}}{\tilde{\sigma}_{p k}+i \omega \epsilon}\right. \\
& \left.\left(1-\exp \left[\gamma_{p k} \Delta x_{k}\right]\right) \prod_{i=k+1}^{j} \exp \left[\gamma_{p i} \Delta x_{l}\right]\right]
\end{aligned}
$$

Solving (47) for $\bar{V}_{j}$ and summing over all $N$ subregions gives the voltage across the total drift region as

$$
\tilde{V}_{d}=\frac{1}{Y_{d}}\left[\tilde{J}_{T}-\tilde{J}_{p 1}(0) \Gamma_{d}\right]
$$

where

$$
\frac{1}{Y_{d}}=\sum_{j=1}^{N} \frac{1-P_{j} \Gamma_{m i}}{Y_{i}}
$$

and

$$
\Gamma_{d}=Y_{d} \sum_{j=1}^{N} \frac{M_{j} \Gamma_{j}}{i \omega C_{j}}
$$

The total drift region impedance is then

$$
Z_{d}=\frac{1}{Y_{d}}\left[1-M_{\mathrm{GH} .1} \Gamma_{d}\right]
$$

Although (63) gives a total device impedance, the real utility of the model lies in the equivalent circuit formulation of Fig. 5, which is easily implemented by a computer-aided design circuit analysis package.

If the analysis of an electron drift region with a cathode injecting at the left is carried out, the expressions and circuits obtained are identical with the analogous hole quantities with the following variable charges: $\tilde{\sigma}_{n}$ is replaced by $\tilde{\sigma}_{n} ; \gamma_{p}$, by $\gamma_{n} ;$ and $\tilde{j}_{p}$, by $\tilde{J}_{n}$. The Gilden-Hines current fraction is redefined as

$$
M_{\mathrm{GH}}=\frac{\tilde{J}_{n}(0)}{\tilde{J}_{T}} .
$$

Much of the earlier analysis of transit-time devices assumed a saturated drift region, i.e. a region with constant carrier velocity and diffusion coefficients. For saturated drift regions a single subregion can be used and the resulting equivalent circuit is shown in Fig. 6 . The analysis presented here reduces directly the results of Kuvås and Lee[21]. In the limit $D_{n . p} \rightarrow 0$ the results further reduce to those of Gilden and Hines [7]. Due to heating of the carriers, the diffusion coefficient in a saturated drift region is generally nonzero. In Fig. 7 a plot of the real part of the impedence for a saturated drift region normalized to the d.c. value $R_{0}=\Delta x^{2} / 2 \epsilon v_{p \text { sat }} A$ is shown for the case of ideal injection, i.e. $M_{\mathrm{GH}}=-1$. (This corresponds to an IMPATT with zero avalanche frequency [7].) Note that a.c. diffusion affects the results even though no d.c. diffusion current exists.

\section{APPLICATION TO THE BARITT DEVICE}

A typical $p^{+} n p^{+}$BARITT electric field profile with the various regions and subregions indicated is provided in Fig. 8. The choice of the number of drift subregions is arbitrary. However, in view of the need of greater resolution in low-field regions a logarithmic scaling is used.

The cathode region equivalent circuit is shown in Fig.

9. This circuit was originally used for BARITT analysis

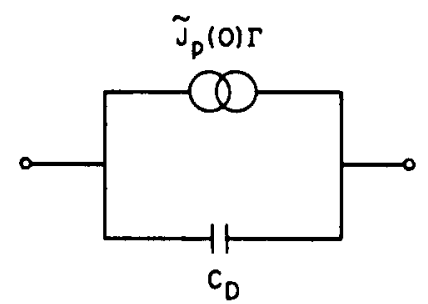

Fig. 6. Equivalent circuit of saturated drift region.

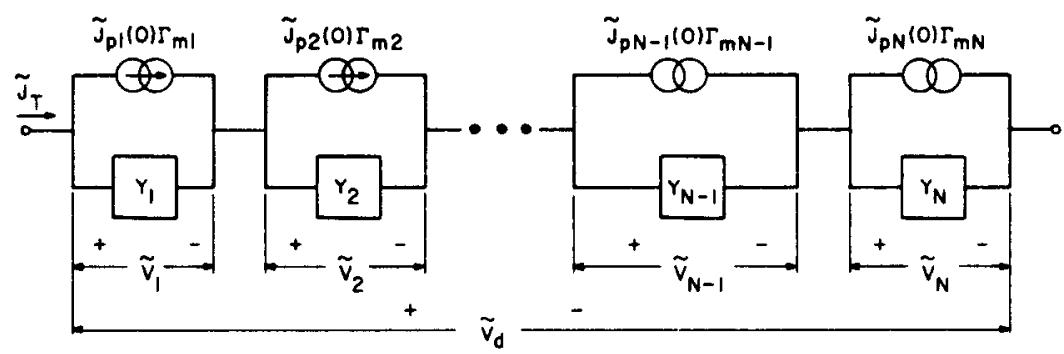

Fig. 5. Equivalent circuit of the drift region. 


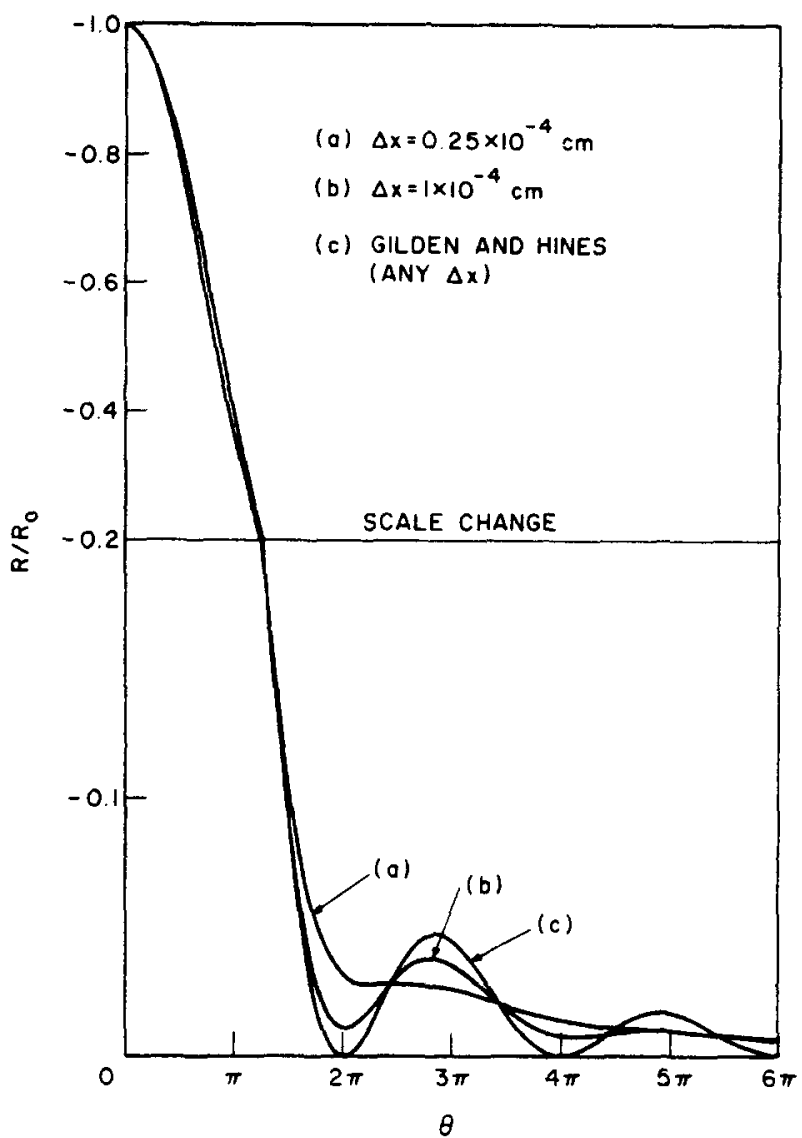

Fig. 7. Small-signal negative resistance of a saturated hole drift region as a function of transit angle $\theta \cdot \theta=\omega \Delta x v_{\text {patu }}$, $M_{\text {OH }}=-1, v_{p \text { sat }}=8 \times 10^{6} \mathrm{cms}^{-1}$. (a) $D_{p}=5 \mathrm{~cm}^{2} \mathrm{~s}^{-1}, \Delta x=2.5 \times 10^{-4} \mathrm{~cm}$. (b) $D_{p}=5 \mathrm{~cm}^{2} \mathrm{~s}^{-1}, \Delta x=1 \times 10^{-4} \mathrm{~cm}$. (c) $D_{p}=0$, any $\Delta x$ (Gilden and Hines theory [7]).

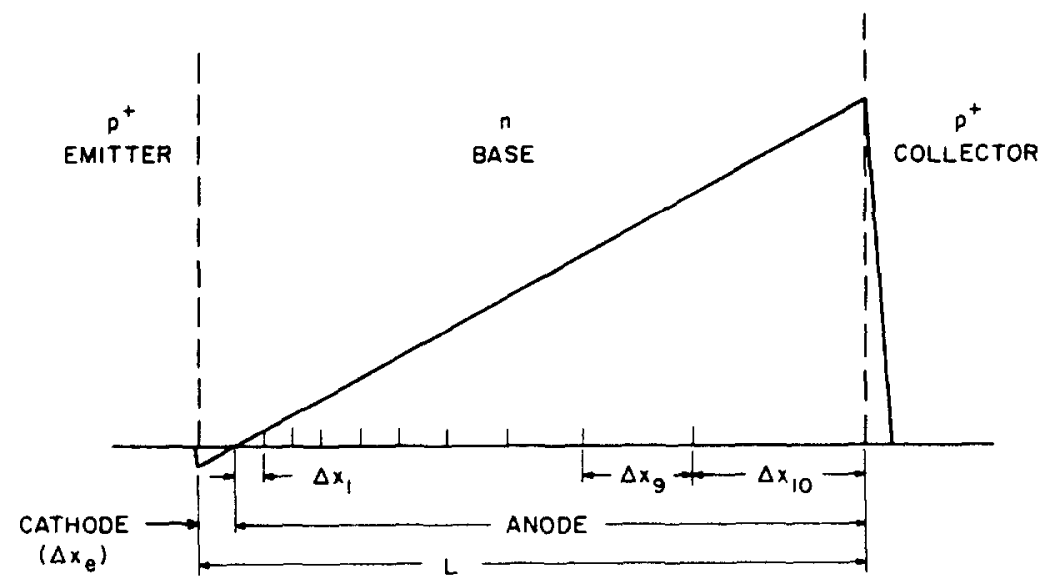

Fig. 8. Electric field profile of a typical $p^{+} n p^{+}$BARITT. $L$ is the base ( $n$-region) length. The drift region is divided logarithmically into 10 subregions. 


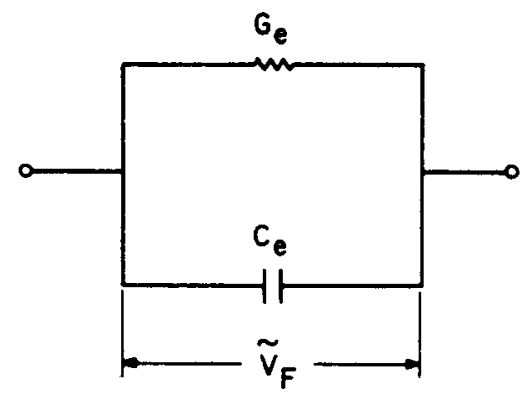

Fig. 9. Cathode region equivalent circuit of a BARITT[8].

by Weller[8]. The cathode circuit parameters are

$$
G_{e}=\frac{I_{\mathrm{d} . \mathrm{c}}}{V_{r}}
$$

and

$$
C_{e}=\frac{\epsilon A}{\Delta x_{e}}
$$

where $\Delta x_{e}$ is the emitter depletion width. The GildenHines current fraction is

$$
M_{\mathrm{GH}}=\frac{G_{e}}{G_{e}+i \omega C_{e}} .
$$

The d.c. solution for the entire device is obtained using the solution method presented in Section 3. The d.c. carrier densities are calculated from the collector metallurgical junction moving toward the zero field point, using the collector boundary condition $p=J_{p} / q v_{\text {sat }}$. The solution, more than a few Angstroms from the junction, is insensitive to the choice of boundary condition. The Trofimenkoff mobility relation (25) was used with the parameters $v_{p \text { sat }}=8 \times 10^{6} \mathrm{~cm} \mathrm{~s}^{-1}$ and $E_{p \text { sat }}=$ $3 \times 10^{4} \mathrm{~V} \mathrm{~cm}^{-1}$.

The calculated small-signal resistance of three devices is plotted as a function of frequency in Fig. 10. There is good agreement between the calculation and the experimental results of Snapp and Weissglas [24]. Since the reactance is largely determined by the depletion capacitance it is not shown.

In Section 2 it was noted that the neglect of energy and momentum relaxation effects prevent the model from being quantitative past the microwave region. While there is no way to include these relaxation processes in a d.c. solution based on (1) and (2), one component can be included in the small-signal model. This is accomplished by the use of a collision time correction in the relationship between the time-varying small-signal velocity and the time-varying small-signal field (eqn (35)). The inclusion of this relaxation time is consistent with the assumed static velocity-field characteristic in the limit $\omega \tau \rightarrow 0$.

The effect of this correction is illustrated in Fig. 11. A Si $p^{+} n p^{+}$BARITT designed for operation at $30 \mathrm{GHz}$ was analyzed both with and without the collision time correction. The effect of the collision time correction is to

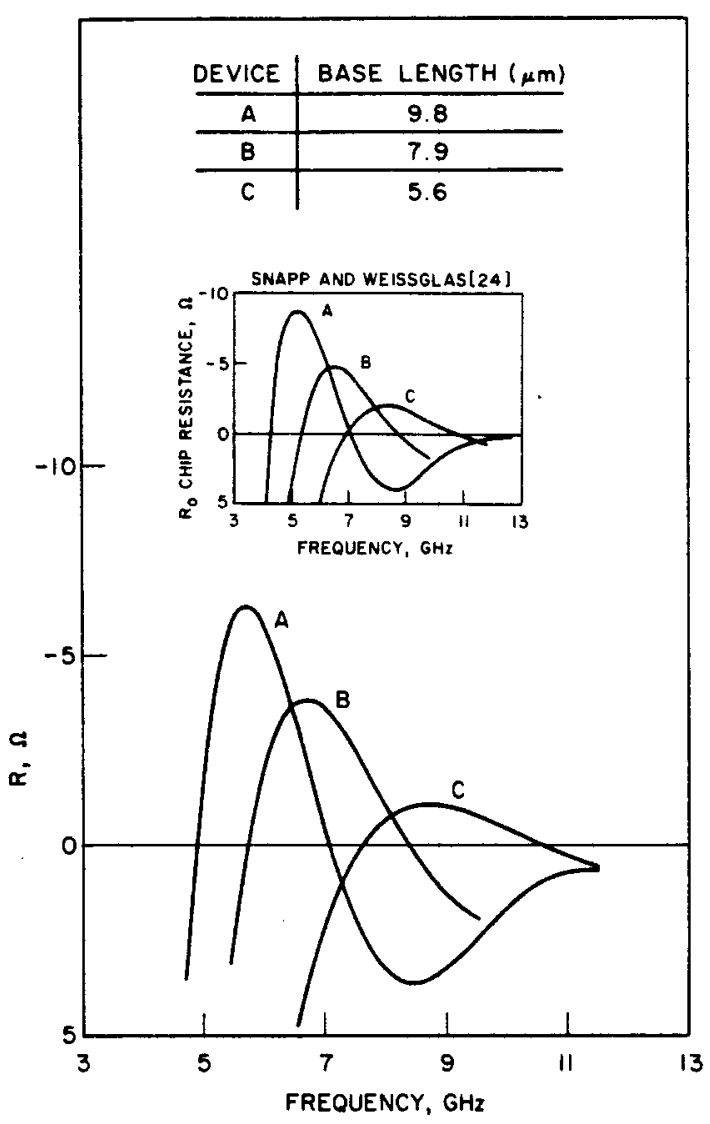

Fig. 10. Comparison of calculated small-signal BARITT resistance with the experimental data of Snapp and Weissglas [24]. $\left(p^{+} n p^{+} \mathrm{Si}, N_{d}=1.2 \times 10^{15} \mathrm{~cm}^{-3}, J_{0}=50 \mathrm{Acm}^{-2}, \mathrm{~A}=3 \times 10^{-4} \mathrm{~cm}^{2}\right)$.

delay the carrier velocity with respect to the field. This increased delay aids a BARITT by reducing the induced current flow in the lossy second quarter of the RF cycle. As a result, the small-signal negative resistance is somewhat larger with the collision time correction.

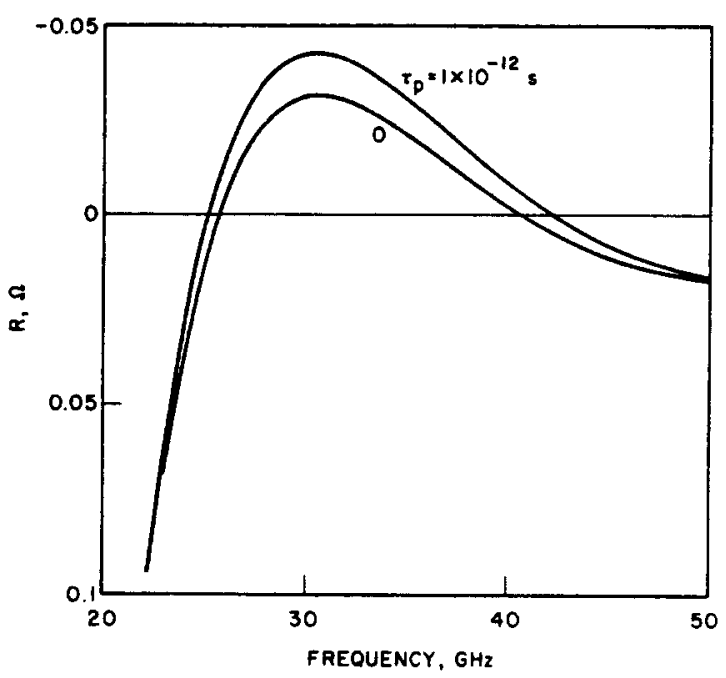

Fig. 11. Comparison of the small-signal resistance of a $K$-band BARITT with and without collision-time corrections to the mobility. $\left(p^{+} n p^{+}, \mathrm{Si}, N_{d}=8 \times 10^{15} \mathrm{~cm}^{-3}, J_{0}=100 \mathrm{Acm}^{-2}, \mathrm{~A}=3 \times\right.$ $\left.10^{-4} \mathrm{~cm}^{2}, L=1.5 \mu \mathrm{m}\right)$. 
It must be stated that inertial effects in the injection process $[25,26]$ have not been included nor have velocity overshoot effects $[12,27]$ been included. Velocity overshoot occurs in the low-field portion of the drift region and will almost certainly degrade BARITT performance. Relaxation effects of this sort are more pronounced if the spatial gradient of the field is large and are therefore more important in the shorter, more highly doped transit-time devices used in the millimeter-wave region. These considerations, along with Fig. 11, suggest that any model based on (1) and (2) is at best qualitative for frequencies in or above the low-millimeter wave region.

An $n^{+} p n^{+}$GaAs BARITT is expected [28] to be a poor microwave source due to the high low-field electron mobility in GaAs which greatly reduces the delay produced by the low-field region. The small-signal resistance of similar $n^{+} p n^{+} \mathrm{Si}$ and GaAs devices is shown in Fig. 12. As expected, the GaAs device exhibits much less negative resistance than the $\mathrm{Si}$ device. The frequency of maximum negative resistance is lower for the GaAs device due to the lower saturated velocity used in the analysis $\left(7.7 \times 10^{6} \mathrm{~cm} \mathrm{~s}^{-1}\right.$ for GaAs vs $1 \times 10^{7} \mathrm{~cm} \mathrm{~s}^{-1}$ for Si). Plots of the d.c. carrier densities in the collector depletion region are shown in Fig. 13.

\section{SUMMARY AND CONCLUSIONS}

An iterative two-carrier d.c. solution for a one-dimensional two-terminal semiconductor device has been presented. This method utilizes an analytic solution for the carrier densities in a region of linearly varying electric field. It can be used in regions of rapidly varying carrier concentration where standard numerical techniques become costly and inaccurate.

A single-carrier small-signal model was also developed for the drift region of a transit-time device under conditions of spatially varying fields and field-dependent diffusion and velocities. The result is expressed in an equivalent circuit which can be easily interpreted in

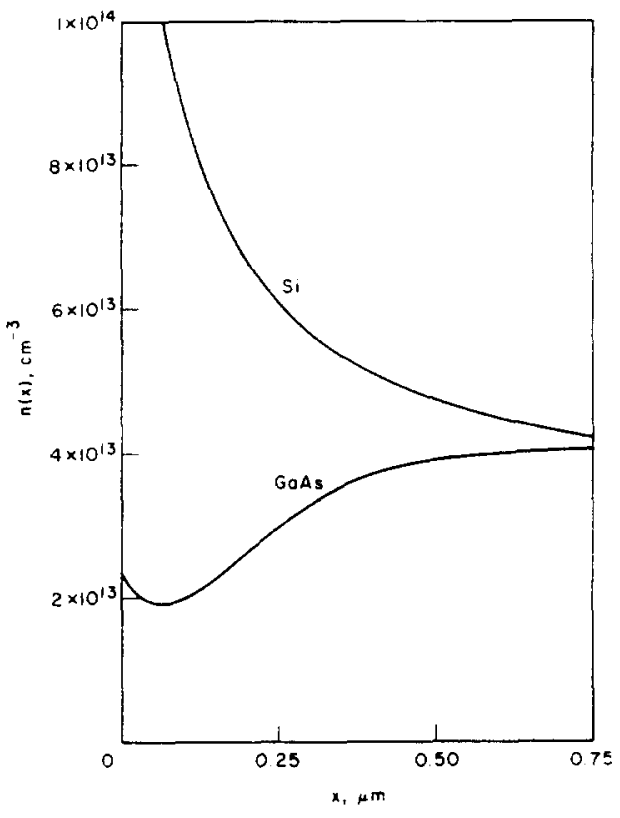

Fig. 13. Collector depletion region d.c. electron densities near the zero field point $(x=0)$ for the devices of Fig. 12. $\left(J_{0}=\right.$ $50 \mathrm{Acm}^{-2}$ ).

terms of the device geometry. This circuit can be conveniently evaluated by a computer-aided design circuit analysis package.

The model was applied to a BARITT device. The d.c. solution converged rapidly. Small-signal results were consistent with published experimental data [24]. As expected[28] $n^{+} p n^{+}$GaAs BARITTs exhibited lower negative resistance than $n^{+} p n^{+} S i$ devices. It was found that a collision-time corrected mobility yielded higher millimeter-wave negative resistances for Si BARITT device drift regions than are predicted by the conventional static model.

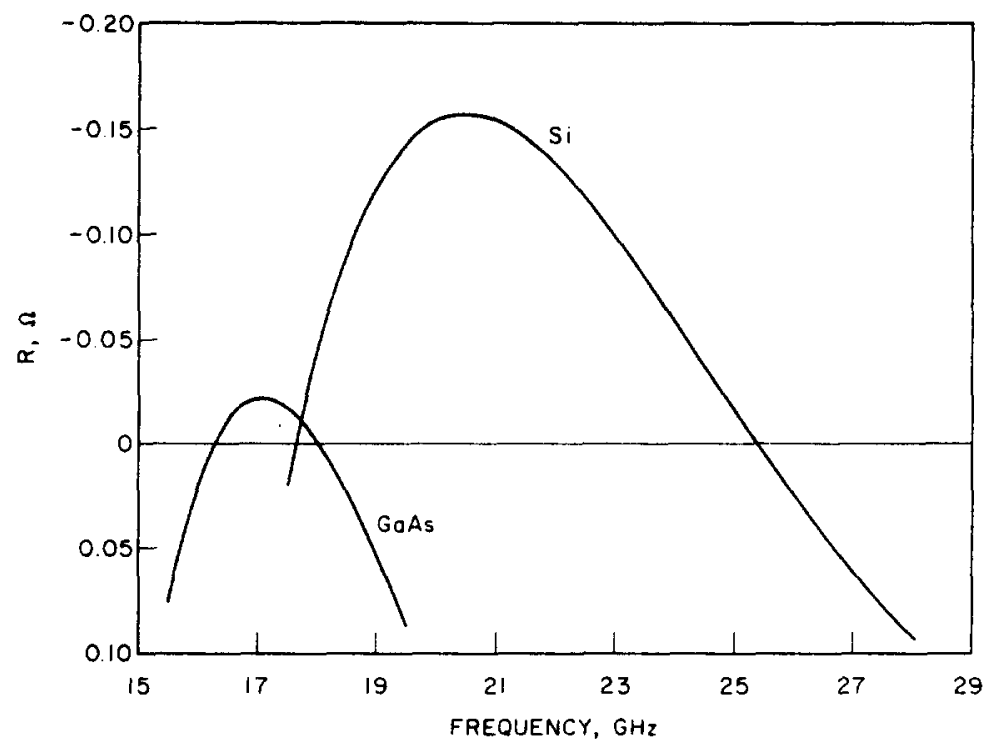

Fig. 12. Small-signal resistance of $n^{+} p n^{+}$GaAs and Si BARRITs, $\left(N_{A}=2.0 \times 10^{15} \mathrm{~cm}^{-3}, J_{0}=50 \mathrm{~A} / \mathrm{cm}^{-2}, \mathrm{~A}=3 \times\right.$ $10^{-4} \mathrm{~cm}^{2}, L=3.6 \times 10^{-4} \mathrm{~cm}$ for both devices). 
Acknowledgements-The authors acknowledge helpful conversations with Drs. Hien Ba Nguyen and Peter A. Blakey and Messrs. Jack R. East and Don E. Peck.

\section{REFERENCES}

1. P. A. Blakey, R. A. Giblin and A. J. Seeds, IEEE Trans. Electron Dev. ED-26. 1718 (1979).

2. P. E. Bauhahn and G. I. Haddad, IEEE Trans. Electron Dev. ED-24. 634 (1977)

3. S. P. Kwok and G. 1. Haddad, Solid-St. Electron. 19. 795 (1976).

4. Semi-Automatic Measurements Using the 8410B Microwave Network Analyzer and the $9825 \mathrm{~A}$ Desk-Top Computer. Hewlett-Packard Application Note 221, May (1978).

5. R. F. Bauer and P. Penfield, Jr., IEEE Trans. Microwave Theory Tech. MTT-22. 282 (1974),

6. W. J. Read, Bell System Tech. J. 37, 401 (1958).

7. M. Gilden and M. E. Hines, IEEE Trans. Electron Dev. ED-13, 169 (1966)

8. K. P. Weller, RCA Rev. 32, 372 (1971).

9. M. E. Elta and G. I. Haddad, IEEE Trans. Electron Dev. ED-25, 694 (1978).

10. J. Christie and J. A. C. Stewart, IEEE Trans. Electron Dev. ED-22, 836 (1975).

11. J. R. Barker and D. K. Ferry, Solid-St. Electron. (to be published).

12. H. Kroemer, Solid-St. Electron. 21, 61 (1978).

13. F. N. Trofimenkoff, Proc. IEEE (Corres.) 53, 1765 (1965).

14. P. J. McCleer and G. I. Haddad, IEEE Trans. Electron Dev. (Corres.) ED-25, 389 (1978).

15. R. A. Kiehl. 1978 Int. Electron Devices Meeting Tech. Digest, pp. 286-289, Washington, DC (1978).

16. J. R. Forrest and A. J. Seeds, 1978 Int. Electron Devices Meeting Tech. Digest, pp. 282-285, Washington, DC (1978).

17. B. Kramer and A. Mircea, Appl. Phys. Lett. 26, 623 (1975).

18. H. Johnson, IEEE Trans. Electron Dev. ED-19, 1156 (1972).

19. Y.C. Wang, Solid-St. Electron. 21, 609 (1978).

20. P. Antognetti, A. Chiabicra and G. R. Bisio, Solid-St. Electron. 16, 345 (1973).

21. R. Kuvås and C. A. Lee, J. Appl. Phys. 41, 3108 (1970).

22. D. E. Snyder, (to be submitted for publication).

23. J. Ziman, Theory of Solids. Cambridge University Press (1972).

24. C. P. Snapp and P. Weissglas, IEEE Trans. Electron Dev. ED-19, 1109 (1972).

25. A. van der Ziel. J. Appl. Phys. 47. 2059 (1976).

26. D. W. Tsang and S. E. Schwarz, J. Appl. Phys. S0, 3459 (1979).

27. T. J. Maloney and J. Frey, J. Appl. Phys. 48, 781 (1977).

28. H. Nguyen-Ba and G. I. Haddad. IEEE Trans. Electron Dev. ED-24, 1154 (1977).

29. M. Abramowitz and I. A. Stegun, Handbook of Mathematical Functions. Dover Publications, New York (1972).

\section{APPENDIX A}

D.c. solution for optical generation

Consider an exponentially varying (optical) generation of the form form

$$
g_{n}=\alpha \Phi_{0} \mathrm{e}^{-\alpha x},
$$

where $\Phi_{0}$ is the incident photon flux and $\alpha$ is the absorption coefficient. The continuity eqn (3) can be integrated to give the electron current as a function of $x$ :

$$
J_{n}(x)=J_{n}(0)-q \Phi_{0}\left(1-\mathrm{e}^{-\alpha x}\right)=J_{n}{ }^{2}+q \Phi_{0} \mathrm{e}^{-\alpha x},
$$

where the new quantity $J_{n}{ }^{*} \triangleq J_{n}(0)-\Phi_{0}$ has been defined. The diffusion coefficient relation used for electrons in $\mathrm{Si}[13]$ is

$$
D=\frac{1}{a_{0}+a_{1}|\bar{E}|}
$$

where $a_{0}=\bar{E}_{n} / v_{n \text { and }}$ and $a_{1}=1 / v_{n .1 .}$. Equation (11) now becomes

$$
\frac{\mathrm{d} N}{\mathrm{~d} x}+\bar{E} N=J_{n}{ }^{\mu}\left(a_{0}+a_{1}|\bar{E}|\right)+q \Phi_{0} \mathrm{e}^{-\alpha x}\left(a_{0}+a_{1}\left|\bar{E}_{1}\right|\right) .
$$

Since (A4) is a linear differential equation, the principal of superposition may be used. The particular solution associated with the first term on the r.h.s. of (A4) is just the d.c. solution (22) previously derived, with $J_{n}{ }^{\prime}$ substituted for $J_{n}$. The particular solution for the second term is found by using the same integrating factor $\exp \left(\xi^{2}\right)$ as before. The particular solution for the second term is then

$$
\begin{aligned}
N_{2}(x)= & \mathrm{e}^{-\xi^{2}}\left[q \Phi _ { 0 } \left(a_{0} \int \mathrm{e}^{\xi^{4}-a x} \mathrm{~d} x\right.\right. \\
& \left.\left.+a_{1} \vee\left(2 \bar{E}_{1}\right) \int|\xi| \mathrm{e}^{\xi^{2}-a x} \mathrm{~d} x+C\right)\right] .
\end{aligned}
$$

When the new variable $\xi^{v}$ is defined as

$$
\xi^{\nu}=\frac{\bar{E}(x)-\alpha}{\sqrt{ }\left(2 \bar{E}_{1}\right)}
$$

and the requirement is added that $\xi^{\nu}$ does not change sign within the linear field region, (A5) becomes

$$
\begin{aligned}
N_{2}(x)= & \mathrm{e}^{-\xi^{2}} q \Phi_{0} \mathrm{e}\left[-\alpha\left(\alpha-2 \bar{E}_{0}\right) / 2 \bar{E}_{1}\right]\left[a_{0} \int \mathrm{e}^{\xi^{\nu^{2}}} \mathrm{~d} x\right. \\
& \left.+a_{1} \sqrt{ }\left(2 \bar{E}_{1}\right) \int|\xi| \mathrm{e}^{\xi^{2}} \mathrm{~d} x+C\right] \\
= & \mathrm{e}^{-\xi^{2}} q \Phi_{0} \exp \left[-\alpha\left(\alpha-2 \bar{E}_{0}\right) / 2 \bar{E}_{1}\right]\left[\left(a_{0}+\alpha a_{1} m^{\nu}\right) \int \mathrm{e}^{\xi^{2}} \mathrm{~d} x\right. \\
& \left.+a_{1} \sqrt{ }\left(2 \bar{E}_{1}\right) m m^{\nu} \int\left|\xi^{\nu}\right| \mathrm{e}^{\xi^{2}} \mathrm{~d} x+C\right] \\
= & \mathrm{e}^{-\xi^{2}} q \Phi_{0} \exp \left[-\alpha\left(\alpha-2 \bar{E}_{0} / 2 \bar{E}_{1}\right]\right. \\
& \times\left[\left(a_{0} m^{\nu}+\alpha a_{1}\right) \vee\left(2 \bar{E}_{1}\right) I_{1}\left(\left|\xi^{\nu}\right|, 0\right)\right. \\
& \left.+2 a_{1} m l_{1}\left(\left|\xi^{\nu}\right|, 1\right)+C\right],
\end{aligned}
$$

where $m=\xi|\xi|$ and $m^{\nu}=\xi^{\nu}|| \xi^{\nu}$. When (A7) is combined with the particular solution for the first term of (A4), the constant $C$ can be evaluated under the boundary condition $N(x=0)=N(0)$ to form the total solution. The hole solution follows similarly.

\section{APPENDIX B}

Properties of integrals $I_{1}$ and $I_{2}$

Solutions are sought for integrals of the form

$$
I_{1}(\eta . n)=\int^{\eta} u^{n} e^{u^{2}} \mathrm{~d} u
$$

and

$$
I_{2}(\eta, n)=\int^{n} u^{n} \mathrm{e}^{-u^{2}} \mathrm{~d} u
$$

where $\eta$ is pure real or pure imaginary. From the preceding definitions of $J_{1}$ and $I_{2}$, it is clear that for a real constant $b$

$$
I_{1}(i b, n)=(i)^{n+1} I_{2}(b, n)
$$

and

$$
I_{2}(i b, n)=(i)^{n+1} I_{1}(b, n) .
$$

The integral functions may be evaluated for imaginary arguments by using the relations (B3) and (B4). It is then necessary to explicitly evaluate $I_{1}$ and $I_{2}$ only for real arguments.

Consider the case of real $\eta$. and $n=0$. The integral function $I_{1}$ becomes

$$
I_{1}(\eta, 0)=\int^{\eta} \mathrm{e}^{u^{2}} \mathrm{~d} u
$$


When the lower limit of the integration is chosen as zero, $I_{1}(\eta, 0)$ and is Dawson's integral function which is well tabulated in [29]. Similarly, when the lower integration limit is chosen as zero. $I_{2}(\eta, 0)$ becomes

$$
I_{2}(\eta, 1)=\frac{1}{2}\left(1-\mathrm{e}^{-\eta^{2}}\right)
$$

$$
I_{2}(\eta, 0)=\int_{0}^{\eta} \mathrm{e}^{-u^{2}} \mathrm{~d} u=\frac{V \pi}{2} \operatorname{erf}(\eta) .
$$

Polynomial approximations for the error function as well as tabulated values appear in [29].

For $n=1$ with real $\eta$, the integral functions are easily evaluated. Taking the lower limit of integration equal to zero yields

Simple recursion relations for higher orders of $n$ are obtained by evaluating (B1) and (B2) once by parts to give

$$
I_{1}(\eta, n)=\frac{1}{2} \eta^{n-1} \mathrm{e}^{\eta^{2}}-\frac{(n-1)}{2} I_{1}(\eta, n-2)
$$

and

$$
I_{1}(\eta+1)=\frac{1}{2}\left(\mathrm{e}^{\eta^{2}}-1\right)
$$

$$
I_{1}(\eta, n)=-\frac{1}{2} \eta^{n-1} e^{-\eta^{2}}+\frac{(n-1)}{2} I_{2}(\eta, n-2)
$$

